\title{
RSSI-based Localization Algorithms using Spatial Diversity in Wireless Sensor Networks
}

\author{
Safa Hamdoun \\ Université Paris-Est \\ LIGM (UMR8049), UPEM \\ F-77454, Marne-la-Vallée France \\ Email: hamdoun@univ-mlv.fr
}

\author{
Abderrezak Rachedi \\ Université Paris-Est \\ LIGM (UMR8049), UPEM \\ F-77454, Marne-la-Vallée France \\ Email: rachedi@univ-mlv.fr
}

\author{
Abderrahim Benslimane \\ French University of Egypt \\ Informatics Research Center (CRI) \\ Cairo, Egypt \\ Email: abderrahim.benslimane@ufe.edu.eg
}

\begin{abstract}
Many localization algorithms in Wireless Sensor Networks (WSNs) are based on received signal strength indication (RSSI). Although they present some advantages in terms of complexity and energy consumption, RSSI values, especially in indoor environments, are very unstable due to fading induced by shadowing effect and multipath propagation.

In this paper, we propose a comparative study of RSSI-based localization algorithms using spatial diversity in WSNs. We consider different kinds of single / multiple antenna systems: Single Input Single Output (SISO) system, Single Input Multiple Output (SIMO) system, Multiple Input Single Output (MISO) system and Multiple Input Multiple Output (MIMO) system. We focus on the well known trilateration and multilateration localization algorithms to evaluate and compare different antenna systems. Exploiting spatial diversity by using multiple antenna systems improve significantly the accuracy of the location estimation. We use three diversity combining techniques at the receiver: Maximal Ratio Combiner (MRC), Equal Gain Combining (EGC) and Selection Combining (SC). The obtained results show that the localization performance in terms of position accuracy is improved when using multiple antennas. Specifically, using multiple antennas at the both sides present better performance than using multiple antennas at the transmitter as well as the receiver side. We also conclude that MRC diversity combining technique outperforms EGC that as well outperforms SC.
\end{abstract}

Index Terms- Wireless Sensor Networks, Indoor localization, Received Signal Strength Indicator, Spatial diversity, Trilateration, Multilateration.

\section{INTRODUCTION}

In recent years, Wireless Sensor Networks (WSNs) have been widely proposed in several applications such as health care, traffic control, environmental monitoring and object tracking [1], [2], [3], [4]. Unfortunately, the exact position of sensors is required to make these variety of applications useful. Accurate localization, thus, remains an interesting area of research.

Several methods based on the received signal strength indication (RSSI) have been proposed in literature. However, RSSI measurements in indoor environments are strongly affected by the propagation environment which lead to bad distance approximations. Exploiting the concept of spatial diversity techniques to improve the accuracy of localization have recently inspired research interest. Spatial diversity, achieved by employing multiple antennas, improve considerably the reliability and the quality of the wireless link [5]. The basic idea consists in providing different copies of the same signal via different paths having undergone different fading. There has been a wide range of research aiming at developing sensors with multiple antennas. Experimental results have been achieved in [6], [7] to show the system requirements and feasibility. Using multiple antennas on the transmitter end (transmit diversity) or the receiver end (receive diversity) leads to a better interpretation of the RSSI values compared with the traditional distance measuring and thus affects the system accuracy.
In general, two types of scenarios can be differentiated in the localization process depending on the direction of the signals being exchanged between the different nodes. Either the target node (node with unknown position), which is possibly attached to a central node with greater processing power, receives signals from the reference nodes called anchors (nodes with known coordinates) or transmits packets to anchors to determine the location estimate. Regarding the scenario considered, receive diversity can be used either by employing multiple antennas under the target node as in [8] or under the reference nodes as in [9]. Authors in [10] investigate the advantage of transmit diversity using multiple antennas under the target node.

In this paper, we assume that only anchor nodes are equipped with multiple antennas. As a consequence, the scenario process in localization will depend on the system model considered. We investigate the advantage of using multiple antennas through three system models: Single Input Multiple Output (SIMO) system where the receive diversity is used, Multiple Input Single Output (MISO) system where the transmit diversity is used and the case of joint receive and transmit diversity called Multiple Input Multiple Output (MIMO) system. We make a comparison relative to the position accuracy among these three system models when using the trilateration as well as the multilateration algorithms. We ground them with sufficient theoretical foundations. Moreover, we use three different methods for 
combining RSSI values at the receiver: Selection Combining (SC), Equal Gain Combining (EGC) and Maximum Ratio Combining (MRC) which are the common linear combining approaches. In order to summarize, the contributions of this paper are:

- A comparative study of the localization performance in terms of average localization errors of the well known trilateration and multilateration localization algorithms when using different kinds of spatial diversity.

- A comparative study of three different diversity combining techniques employed at the receiver on localization performance: SC, MRC and EGC.

The rest of this paper is organised as follows. In Section II, related work is presented, while in Section III, the localization process under different system models are proposed. In Section IV, we present and discuss our results. Finally, we conclude our work in section V.

\section{RELATED WORK}

\section{A. localization}

In WSNs, localization protocols can be classified into two main categories regarding the mechanism used for determining the position of nodes: range-based and range-free protocols. Range based techniques depends on measurements to calculate either the distances or the angles between nodes which require introducing extra hardware. In the second class, the location of the target node is estimated relying on hypothesis about the network connectivity without the need of additional hardware.

Several algorithms belonging to the range free context have been proposed. In [11], a proximity based protocol called Centroid algorithm is proposed. The nodes at known positions transmit beacon signals periodically to neighbours. The listener node, using this proximity information, estimates its position using the centroid model. In [12], a novel method called Approximate Point In Triangle (APIT) was introduced. The unknown node tests whether it is inside the triangles formed by connecting three between the audible reference nodes. This test is repeated for various reference node combinations. The estimated position is the centre of gravity of the intersection of all of the triangles in which a node reside.

Concerning range-based localization, many ranging technologies are possible. Time based localization methods like Time of Arrival (ToA) or Time Difference of Arrival (TDoA) have been widely proposed [13], [14]. In ToA, the unknown node and the receivers must be synchronised to estimate the distance via signal propagation time. While in TDoA, the synchronization of the unknown node is not required, since the method operates on the difference of arrival times.

Although both ToA and TDoA are proved to achieve a good accuracy in [15], these techniques present an expensive and energy consuming localization. Received Signal Strength Indicator (RSSI) technology has been proposed as a cost effective solution [16], [17], [18]. In RSSI techniques, models are used to estimate distance through signal strength. However, distance estimation in RF based methods are degraded due to shadowing and multipath effects.

In this paper, we consider RSSI since it is advantageous in terms of cost and energy consumption despite the large variations of its measurements caused by multipath fading as well as shadowing in indoor environments. Various enhancement schemes have been proposed in order improve the accuracy of nodes with unknown position. Authors in [19] present a new method by defining preprocessing steps to optimize and calibrate the experimental data before beginning the positioning procedure. In [20], authors show the impact of anchor placement on localization performances. Recently, many researchers exploit the concept of spatial diversity and investigate its impact on localization accuracy. In the next subsection, we will explain the concept of spatial diversity and we will present some works showing its impact on position accuracy.

\section{B. Spatial diversity}

Diversity techniques are a common approach that help mitigating the degrading effects of fading. Different types of diversity are usually used in wireless communication such as time diversity, frequency diversity and spatial diversity. Spatial diversity is the most attractive since additional resources in the wireless link are not required. The concept behind spatial diversity is relatively simple: the receiver is provided multiple copies of the transmitted signal via different paths so that they will undergo independent fading.

1) SISO: The simplest form of a communication link is the Single Input Single Output (SISO) system. Both the transmitter and the receiver are equipped with a single antenna as depicted in figure 1. Spatial diversity in this case can not be used. This model is introduced for comparison purpose to show the clear advantage of using spatial diversity on system performances.
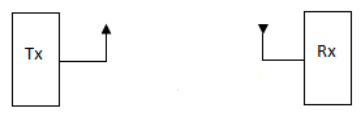

Fig. 1: SISO system: Single Input Single Output

The wireless channel is modelled with the equation

$$
y=h x+n
$$

where $\mathrm{h}$ and $\mathrm{n}$ represents the fading and noise, respectively . Due to fading, the reliability of the information extracted from the received signal, manifested through the error probability, is poor. The Bit Error Rate (BER) can be defined in terms of the probability of error $\left(P_{e}\right)$. We show how the corresponding error probability for SISO system is critically damaged by 
fading. The error probability satisfies [21],

$$
P_{e} \leq \exp -\frac{\left|h^{2}\right| S N R}{2}=\frac{1}{1+\frac{S N R}{2}}
$$

Where SNR is the signal-to-noise ratio.

2) SIMO: The Single Input Multiple Output (SIMO) system, also known as receive diversity is depicted in figure 2 . The
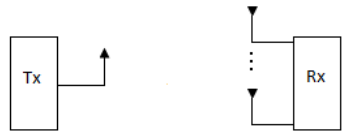

Fig. 2: SIMO system: Single Input Multiple Output

transmitter is equipped with a single antenna and the receiver has multiple antennas. In this case, the receiver is provided a number of independent copies of the transmitted signal to overcome the effects of fading. Let $\mathrm{N}$ be the number of receive antennas. The signal received in antenna $i$ is given by

$$
y_{i}=h_{i} x+n_{i}, i=1,2, \ldots, N
$$

where $h_{i}$ and $n_{i}$ are the fading and noise, respectively, as experienced by antenna $\mathrm{i}$. We assume the fading is independent, which is the case, provided the antennas are sufficiently spaced from each other. The error probability achieved in this case satisfies,

$$
P_{e} \leq \exp \left\{-\frac{S N R \sum_{i=1}^{N}\left|h_{i}\right|^{2}}{2}\right\}=\frac{1}{\left\langle 1+\frac{S N R}{2}\right\rangle^{N}}
$$

We can conclude that the error probability is much smaller than the one corresponding to the SISO system, in which no spatial diversity exists.

Different diversity combining techniques can be used at the receiver. The common linear combining methods are: Selection Combining (SC), Equal Gain Combining (EGC) and Maximal Ratio Combining (MRC). The receiver in SC technique selects the best signal from the different antennas. In EGC, all the received signals are co-phased at the receiver and added together, whereas in MRC, the signals from each channel are weighted and added together. The performance improvement in terms of BER is maximum for Maximal Ratio Combining (MRC), while Equal Gain Combining(EGC) and Selective Combining provide inferior performances [22].

3) MISO: The Multiple Input Single Output (MISO) communication model also known as transmit diversity employ multiple antennas at the transmitter and a single antenna at the receiver as depicted in figure 3. Compared with SIMO system, the processing is moved from the receiver to the transmitter. The total transmit power is divided amongst all antennas. Let $\mathrm{M}$ be the number of transmit antennas. The received signal is given by

$$
y=\sum_{j=1}^{M} h_{j} x_{j}+n
$$

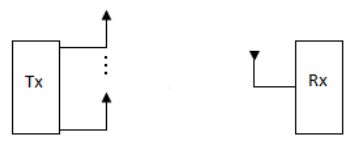

Fig. 3: MISO system: Multiple Input Single Output

where $h_{j}$ is the fading corresponding to transmit antenna $\mathbf{j}$ and $x_{j}$ is the symbol sent through antenna $\mathrm{j}$.

The error probability satisfies,

$$
P_{e} \leq \frac{1}{\left\langle 1+\frac{S N R}{2}\right\rangle^{M}}
$$

4) MIMO: In Multiple Input Multiple Output (MIMO) system, multiple antennas are deployed on both the transmitter and the receiver as illustrated in figure 4 . Let $\mathrm{N}$ and $\mathrm{M}$ be the
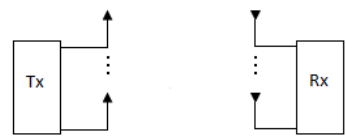

Fig. 4: MIMO system: Multiple Input Multiple Output

number of receive and transmit antennas, respectively. The received signal at antenna $i$ will be

$$
y_{i}=\sum_{j=1}^{M} h_{i j} x_{j}+n_{i}, i=1,2, \ldots, N
$$

The error probability satisfies,

$$
P_{e} \leq \frac{1}{\left(1+\frac{S N R}{2 \min \{N, M\}}\right)^{N M}}
$$

\section{C. localization exploiting spatial diversity}

Recently, many research studies have introduced the concept of spatial diversity in localization and showed its impact on location estimate. For instance, authors in [9] provide an experimental evaluation of multiple receive antennas on anchor nodes on both 802.11 test bed as well as 802.15.4 test bed. Diverse set of algorithms ranging from nearest neighbour, statistical maximum likelihood estimation and multilateration were used. Various simple antenna combinations schemes were considered. They assume averaging or not averaging the data from multiple antennas depending on their coordinates. Results show that averaging or not RSS values depends on the distance between the antennas and the distance between the testing points. The performance of localization algorithms in nearly all cases improved when using multiple antennas. Specifically, the median and 90th percentile error can be reduced up to $70 \%$. In [8], authors investigate the advantage of using multiple receive antennas on target nodes for three 
algorithms (Min-Max, Maximum Likelihood, Trilateration). Two antennas spaced $10 \mathrm{~cm}$ far from each other were used and the average of the two RSSI values is considered as the input for the localization algorithms. Experimental results prove an average improvement in the accuracy by $20 \%$. A different approach in [10] suggest using multiple transmit antennas on target nodes and selecting one out of them in a round robin manner. The Maximum Likelihood location estimation method was used. Experimental results show an improvement in the location accuracy performance by around $20 \%, 27 \%$ and $40 \%$ for the case of two, three, and four antennas, respectively. Using multiple antennas will improve localization accuracy since diversity is used.

In this study, we assume multiple antennas on anchor nodes rather than target nodes since these latters may be limited by size, cost and battery drain. According to this consideration, either transmit or receive diversity will be used. We also consider the case of multiple antennas under both target and anchor nodes. A comparative study of the performance in terms of localization error metric of localization algorithms namely trilateration and multilateration under different system models will be made. We also show the impact of different diversity combining techniques employed at the receiver on position accuracy namely SC, EGC and MRC.

\section{LOCALIZATION UNDER DIFFERENT SYSTEM MODELS}

Usually, the localization process in RF based techniques is divided into two phases. During the first phase, range measurements between the unknown node and the reference nodes are calculated. While in the second phase, a location estimate phase using geometric principles such as trilateration and multirateration is applied. We will focus on trilateration as well as multilateration geometry based localization techniques using RSSI positioning technology. In order to estimate the distance between the target and each reference node, a relationship between the received signal power and distance is used. We apply the Rappaport propagation model with the combined effect of path loss and shadowing [23]. In indoor environments, this propagation model is mainly used and is given by

$$
P_{r}=P_{t}+20 \log \left(\frac{\lambda}{4 \pi d_{0}}\right)-10 n \log \left(\frac{d}{d_{0}}\right)+\psi_{d B}
$$

Where $\lambda$ is the wavelength, $d_{0}$ is a reference distance, $\mathrm{d}$ is the transmitter-receiver distance, $\mathrm{n}$ is the path loss exponent, $\psi_{d B}$ is is a zero-mean Gaussian random variable, and $P_{t}$ and $P_{r}$ are the transmitter and receiver powers in $\mathrm{dB}$. In multilateration, the distance estimate is used to generate a circle around each reference node on which the target node must be. The position estimate of a node is given by the intersection of these circles. This technique is called trilateration when using three reference nodes. Authors in [24] have proved that the accuracy of the RSS ranging is improved and an accurate localization is achieved when reducing the Bit Error Rate (BER). While they have proposed a localization algorithm that employs a new coding method to reduce the BER, we use spatial diversity as we have showed its impact on the BER.

In this section, we present the different system models considered when using the trilateration and multilateration algorithms in order to investigate their impact on position accuracy.

\section{A. Localization under SISO model}

We consider SISO model for comparison purpose where both target and anchor nodes are equipped with a single antenna. Each anchor node (receiver) collects the signal strength of the target node in order to calculate the ranges between the transmitter and receiver. Figure 5 illustrates the trilateration algorithm considering the basic model, SISO model.

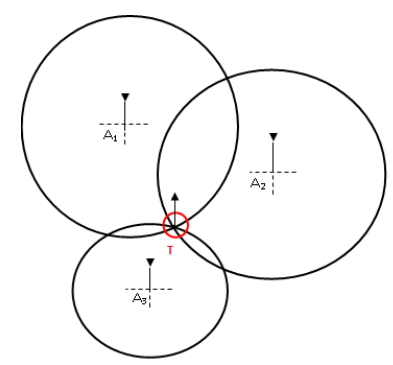

Fig. 5: Trilateration algorithm using SISO model

\section{B. Localization under SIMO model}

We consider SIMO model also known as receive diversity where the target node, being the transmitter, is equipped with a single antenna and anchor nodes (receivers) are equipped with multiple antennas. Each anchor node collects the RSSI measurements in order to calculate the ranges between the transmitter and receiver. Figure 6 illustrates the trilateration algorithm considering the SIMO model.

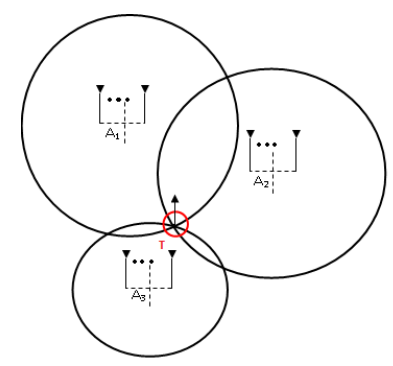

Fig. 6: Trilateration algorithm using SIMO model

The algorithm flow chart for localization under SIMO model is shown in figure 7. First, anchor and target nodes are dislocated (figure 12). During the range measurements phase, RSSI values are computed for each receive antenna taking into account the shadowing effect. The estimate distance between the target and each anchor is calculated using the 
Rappaport propagation model. Finally, the estimate target position is determined using trilateration and multilateration localization methods. The performance of the localization algorithms is determined in terms of average localization errors. This latter is defined as the difference between estimate coordinates and real ones. This procedure is repeated 100 times and the average of the measurements is computed. We use three diversity combining techniques at the receiver: SC, EGC and MRC techniques. If there are $\mathrm{N}$ antennas, and the RSSI value received from antenna $\mathrm{i}$ is $R_{i}$, then we combine these values as following [24],

Selection Combining method: It picks the maximum RSSI measurement among all the branches, i.e.,

$$
R_{\max }=\max \left\{R_{1}, \ldots, R_{N}\right\}
$$

Equal Gain Combining method: All the RSSI measurements are averaged, i.e.,

$$
R_{a v g}=\frac{1}{N} \sum_{i=1}^{N} R_{i}
$$

Maximum Ratio Combining method: The RSSI measurements are combined in the following way

$$
R_{m r c}=\frac{1}{\sum_{i=1}^{N} R_{i}} \sum_{i=1}^{N} R_{i}^{2}
$$

\section{Localization under MISO model}

We consider MISO model known as transmit diversity. The target node, being a receiver, is equipped with a single antenna and anchor nodes or transmitters are equipped with multiple antennas. In this case, the target node gathers the RSSI measurements from each reference node to calculate the transmitter-receiver distance. Figure 8 illustrates the trilateration algorithm considering the MISO model. The algorithm flow chart for localization under MISO model is depicted in figure 9. The total transmit power is divided among transmit antennas.

\section{Localization under MIMO model}

We consider the case of MIMO system where multiple antennas can be used on both anchor and target nodes, as illustrated in figure 10. The algorithm flow chart for localization under MIMO model is depicted in figure 11. In this case, transmit diversity as well as receive diversity are jointly used.

\section{PERFORMANCE EVALUATION}

\section{A. Description of the simulation environment}

For performance evaluation of localization algorithms, we used a square room of size $20 \mathrm{~m} \times 20 \mathrm{~m}$. We configured three and four anchor nodes for trilateration and multilateration algorithms, respectively as depicted in figure 12 . We chose for both anchor deployments, a position P1 of the target which is equally distant from reference nodes and two other different

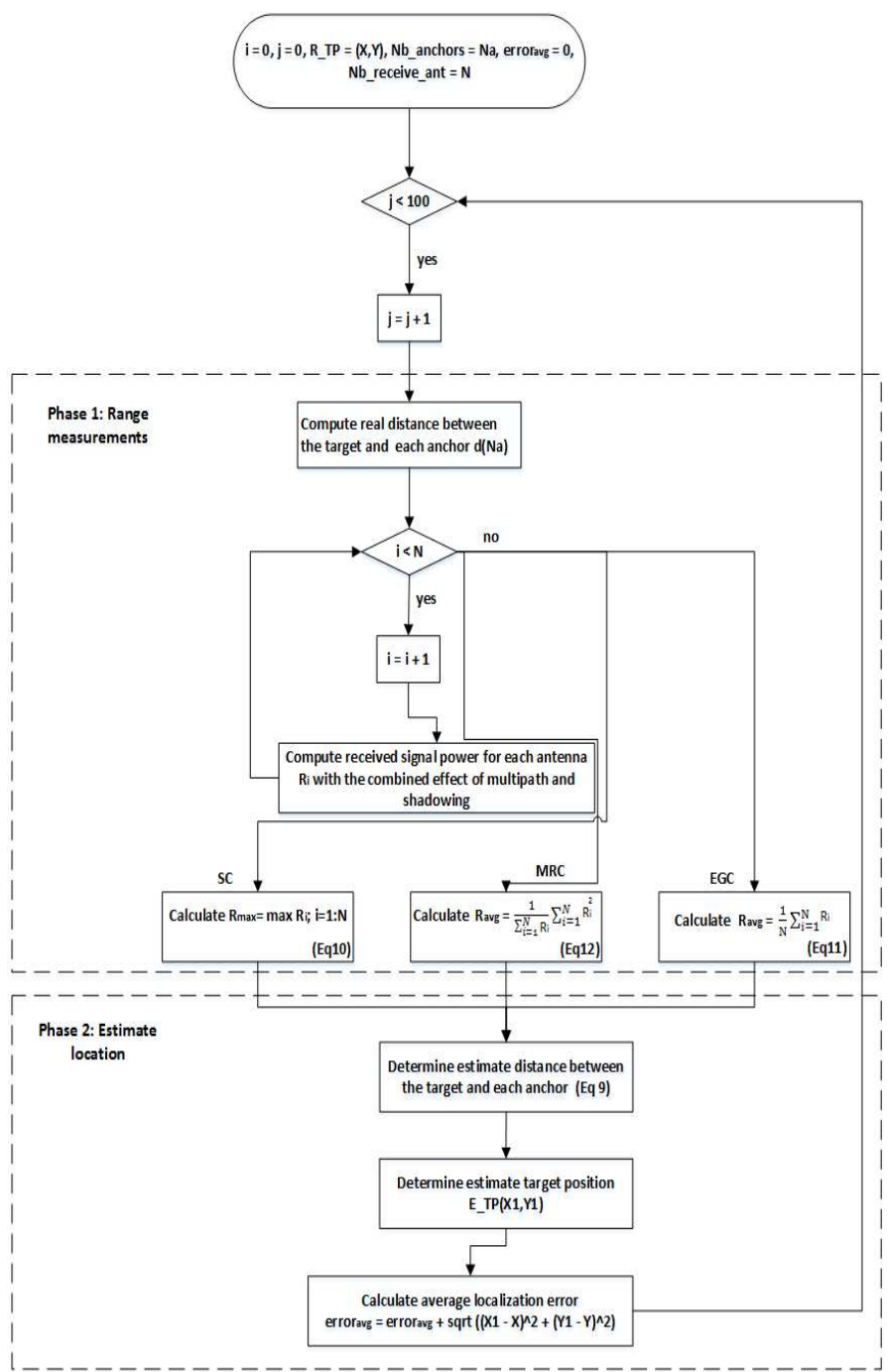

Fig. 7: Flowchart of the localization process under SIMO model

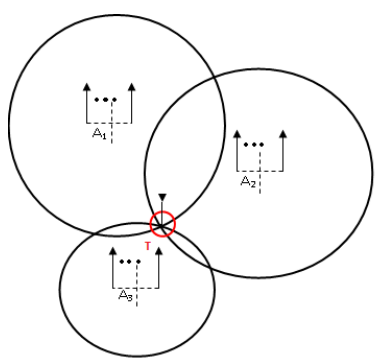

Fig. 8: Trilateration algorithm using MISO model

target's positions which have different distances from P1. The frequency used is equal to $900 \mathrm{Mhz}$. Simulation was done using the software Matlab. For receive diversity, we used two receive antennas and one transmit antenna $(1 \times 2)$, while for transmit diversity two transmit antennas and one one receive antenna are used $(2 \times 1)$. Concerning the joint transmit receive 


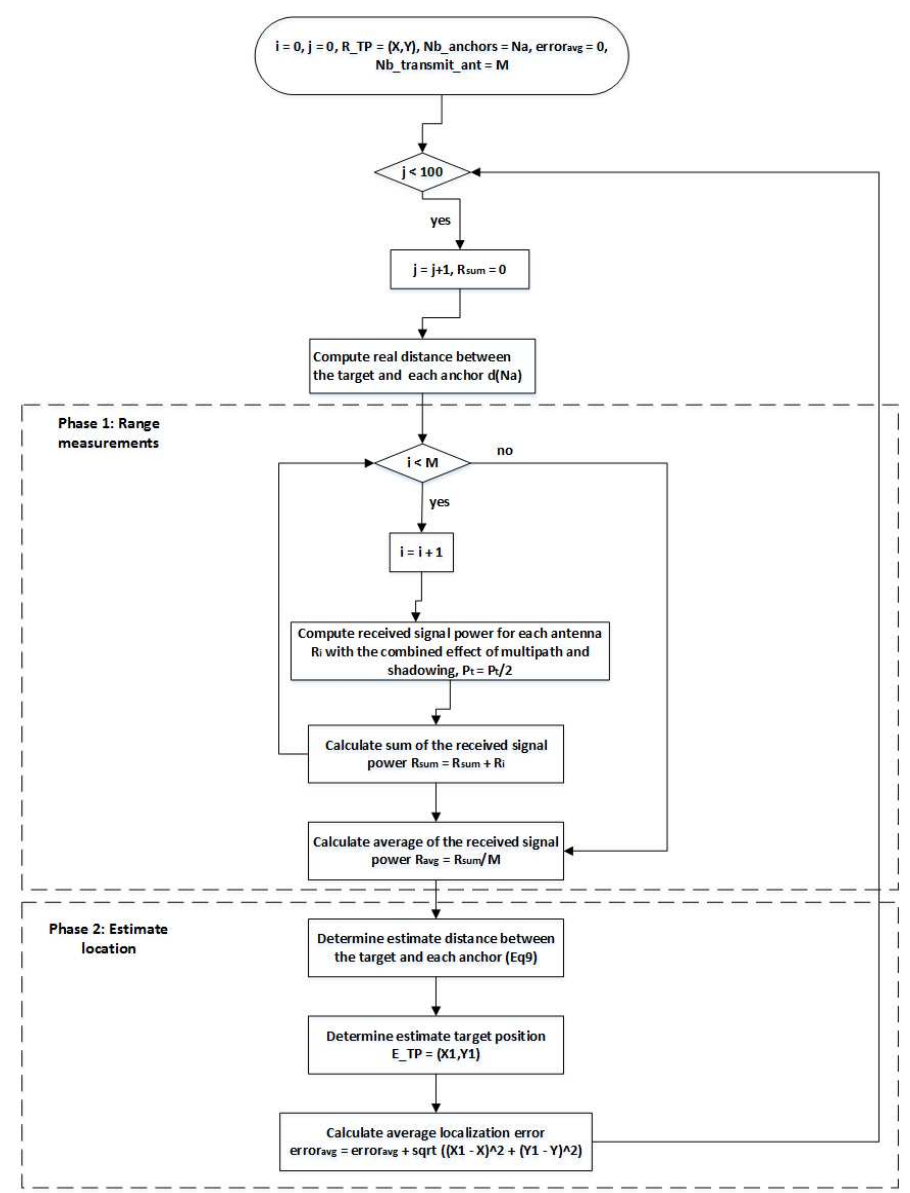

Fig. 9: Flowchart of the localization process under MISO model

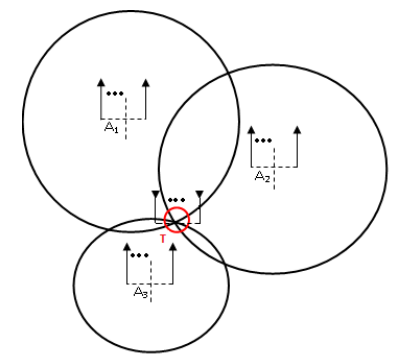

Fig. 10: Trilateration algorithm using MIMO model

diversity, we used two antennas at the both sides $(2 \times 2)$. We used the localization error metric which is defined previously in order to characterize the performance of the localization algorithms.

\section{B. Results and discussions}

In figures 13 and 14, the average localization error of the multilateration and trilateration algorithms respectively is evaluated against the shadowing standard deviation when using different system models with different target positions. To simulate different indoor environments, the standard

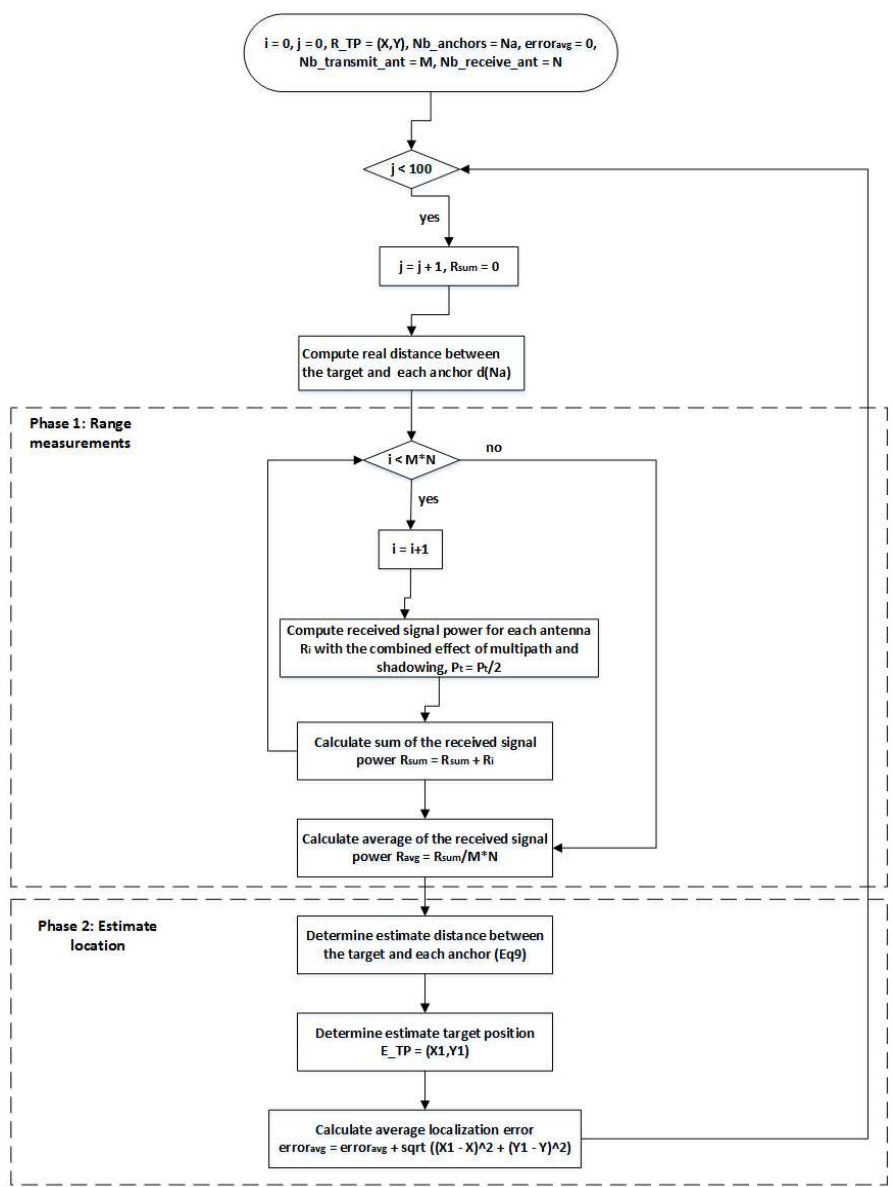

Fig. 11: Flowchart of the localization process under MIMO model

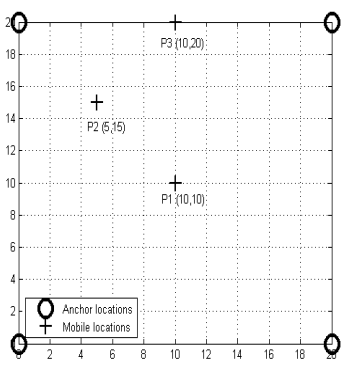

(a) Multilateration

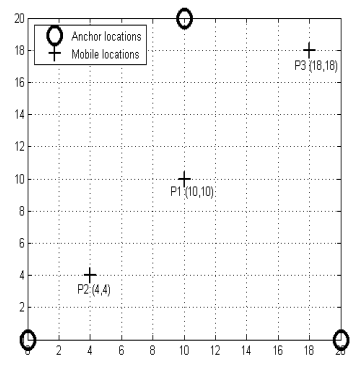

(b) Trilateration
Fig. 12: Position of the nodes

deviation of the shadow fading was changed from $1 \mathrm{~dB}$ to $6 \mathrm{~dB}$. The higher the shadowing standard deviation, the worser is the performance of both localization algorithms in terms of average localization errors. The good performance of MIMO over SIMO, and MISO systems is attributed to the higher number of signal copies at the receiver having undergone different fading. The SISO system present the worse performance which is expected since the diversity 


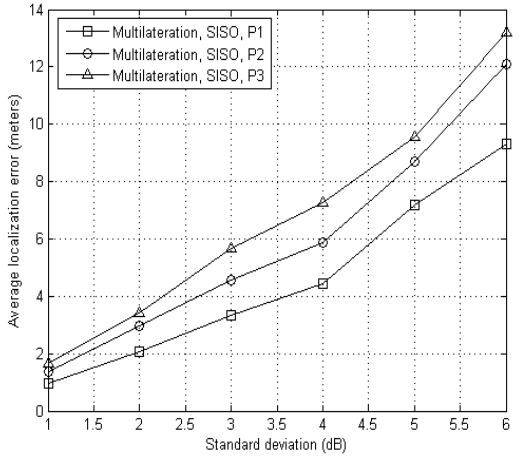

(a) Multilateration algorithm with SISO model

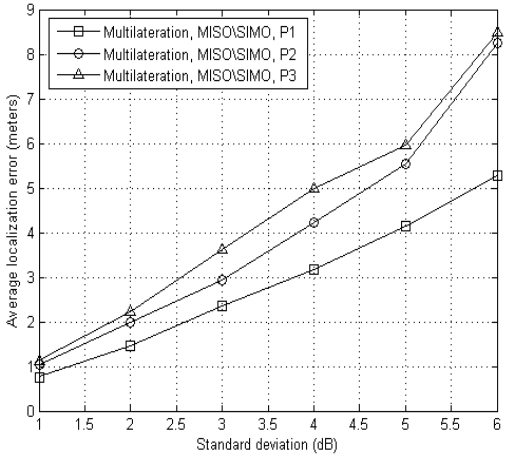

(b) Multilateration algorithm with SIMO and MISO models

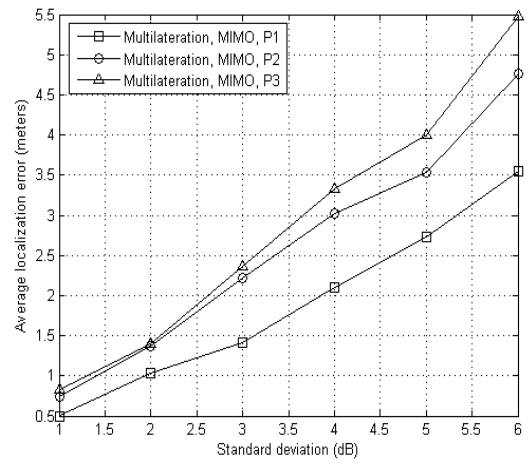

(c) Multilateration algorithm with MIMO model

Fig. 13: Multilateration algorithm under different system models

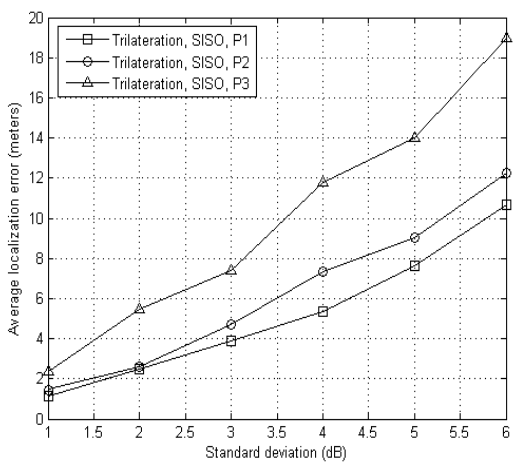

(a) Trilateration algorithm with SISO model

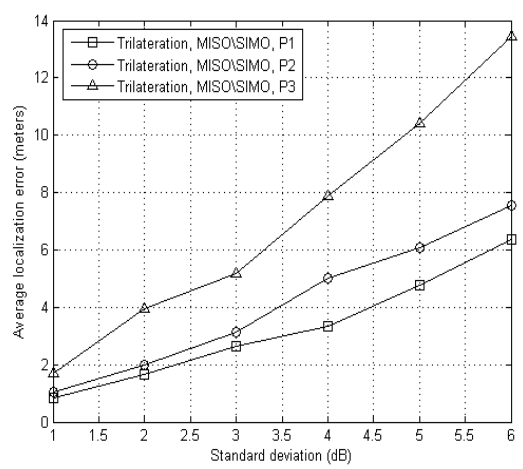

(b) Trilateration algorithm with SIMO and MISO models

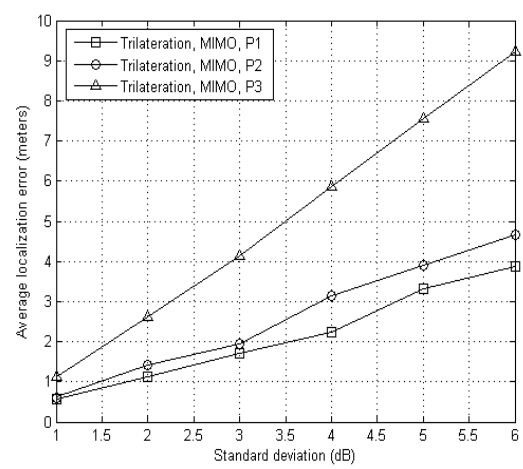

(c) Trilateration algorithm with MIMO model

Fig. 14: Trilateration algorithm under different system models

gains is not exploited. Similar performance in terms of average localization errors for each of the localization algorithms under SIMO and MISO models is obtained. This is understandable since the processing is moved from the receiver to the transmitter.

Amongst the three positions of the target, the optimal results were obtained for the position P1 for both anchor nodes deployment. On the other hand, the localization accuracy with the target position P2 outperforms P1. Indeed, the closer the target node to the center of gravity, the better the results are.

Figure 15 shows the impact of the number of antennas used at the receiver on the average localization error obtained in meters. The target position used is $\mathrm{P} 1$ for both localization algorithms and the shadow fading standard deviation is assumed to be $3 \mathrm{~dB}$. An improvement in the performance of about $30 \%$ is achieved when using four antennas comparing to the case where two antennas are used. Thus, the performance accuracy improves considerably while increasing the number of antennas. However, this benefit comes at the expense of system complexity. Beyond the number of ten antennas, a little improvement is achieved.

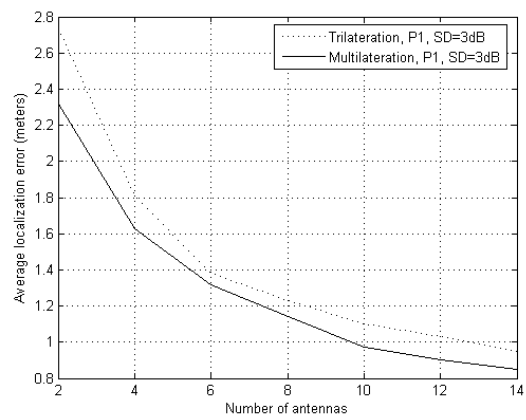

Fig. 15: Localization error when varying the number of antennas

In figure 16, the average localization errors using multilateration and trilateration algorithms is measured against the shadow fading standard deviation. For comparison purpose, we use the same position of the target P1 for both algorithms. Better performance are obtained when using the multilatera- 
tion algorithm compared to the trilateraton one. Indeed, the localization accuracy is higher when more anchors are used. The difference in terms of position accuracy between both algorithms is accentuated from $4.5 \mathrm{~dB}$. This is attributed to the random shadowing effect where its variability is larger at higher standard deviation.

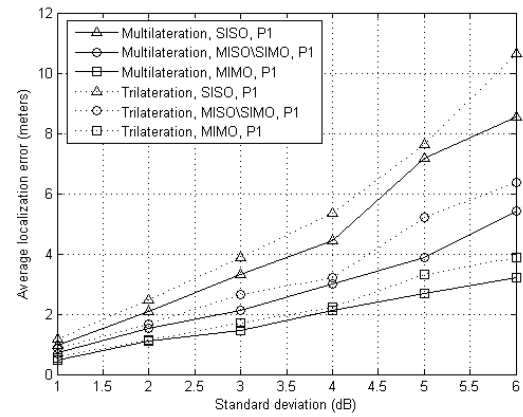

Fig. 16: Localization error when using the trilateration and multilateration algorithms

In figure 17, a comparison of the average localization errors using multilateration algorithm considering three different methods for combining RSSI values at the receiver: $\mathrm{SC}$, EGC and MRC is illustrated. We can observe that the accuracy is the highest for MRC technique and the lowest for $\mathrm{SC}$ technique, with EGC performance closer to MRC one. Although it is known that the maximal ratio combining is the optimal linear combining technique, the receiver is more complex since it is dependent on the number of paths available at the receiver. Also, EGC has the same feature in terms of receiver complexity. $\mathrm{SC}$ is a suboptimal combining scheme that alleviate the complexity at the receiver but provides worse performance in terms of position accuracy.

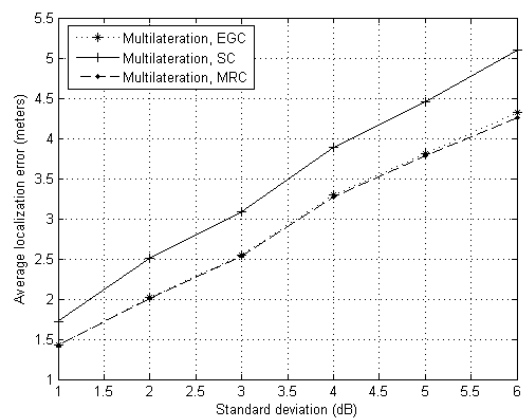

Fig. 17: Localization error under SIMO model when using MRC, EGC and SC

\section{CONCLUSIONS}

In this paper, we studied the impact of different kinds of spatial diversity on localization accuracy in indoor environments when varying the shadowing effect. We used the multilateration as well as the trilateration algorithms, based on RSSI values, to estimate the target position. Three system models illustrating the spatial diversity were considered: transmit diversity (MISO), receive diversity (SIMO) and the joint transmit-receive diversity (MIMO). We found that the localization accuracy is improved compared to the single antenna system (SISO). Specifically, MIMO system performs SIMO and MISO systems which in turn present similar performance. We showed that the multilateration algorithm present better results compared with the trilateration one. We also compared the average localization error using different diversity combining methods at the receiver, namely, SC, MRC and EGC. We found that MRC performs the best and that SC is the worst although this latter is the simplest in terms of implementation. As future work, we plan to evaluate these models by using a real experimentation platform.

\section{REFERENCES}

[1] JeongGil Ko, Chenyang Lu, Mani B Srivastava, John A Stankovic, Andreas Terzis, and Matt Welsh. Wireless sensor networks for healthcare. Proceedings of the IEEE, 98(11):1947-1960, 2010.

[2] Chen Wenjie, Chen Lifeng, Chen Zhanglong, and Tu Shiliang. A realtime dynamic traffic control system based on wireless sensor network. pages 258-264, 2005.

[3] Guillermo Barrenetxea, François Ingelrest, Gunnar Schaefer, and Martin Vetterli. Wireless sensor networks for environmental monitoring: the sensorscope experience. pages 98-101, 2008.

[4] Ibtissem Boulanouar, Stéphane Lohier, Abderrezak Rachedi, and Gilles Roussel. Cta: A collaborative tracking algorithm in wireless sensor networks. pages 529-534, 2013.

[5] Nitika Sachdeva and Deepak Sharma. Diversity: A fading reduction technique. International Journal of Advanced Research in Computer Science and Software Engineering, ISSN, 2012.

[6] Mubashir Husain Rehmani, Thierry Alves, Stéphane Lohier, Abderrezak Rachedi, and Benoit Poussot. Towards intelligent antenna selection in ieee 802.15. 4 wireless sensor networks, 2012

[7] Husain Rehmani Mubashir, Abderrezak Rachedi, Stéphane Lohier, Thierry Alves, Benoit Poussot, et al. On the feasibility of making intelligent antenna selection decision in ieee 802.15. 4 wireless sensor networks. pages 1-6, 2013.

[8] Emanuele Goldoni, Alberto Savioli, Marco Risi, and Paolo Gamba. Experimental analysis of rssi-based indoor localization with ieee 802.15 4. pages 71-77, 2010.

[9] Konstantinos Kleisouris, Yingying Chen, Jie Yang, and Richard P Martin. Empirical evaluation of wireless localization when using multiple antennas. Parallel and Distributed Systems, IEEE Transactions on, 21(11):1595-1610, 2010.

[10] Shinsuke Hara and Daisuke Anzai. Three estimation methods for rssibased localization with multiple transmit antennas. pages 1-5, 2007.

[11] Nirupama Bulusu, John Heidemann, and Deborah Estrin. Gps-less lowcost outdoor localization for very small devices. Personal Communications, IEEE, 7(5):28-34, 2000.

[12] Tian He, Chengdu Huang, Brian M Blum, John A Stankovic, and Tarek Abdelzaher. Range-free localization schemes for large scale sensor networks. pages 81-95, 2003.

[13] Yiu-Tong Chan, Wing-Yue Tsui, Hing-Cheung So, and Pak-chung Ching. Time-of-arrival based localization under nlos conditions. Vehicular Technology, IEEE Transactions on, 55(1):17-24, 2006.

[14] KC Ho and YT Chan. Solution and performance analysis of geolocation by tdoa. Aerospace and Electronic Systems, IEEE Transactions on, 29(4):1311-1322, 1993.

[15] Kegen Yu, Jean-philippe Montillet, Alberto Rabbachin, Paul Cheong, and Ian Oppermann. Uwb location and tracking for wireless embedded networks. Signal Processing, 86(9):2153-2171, 2006.

[16] Cesare Alippi and Giovanni Vanini. A rssi-based and calibrated centralized localization technique for wireless sensor networks. pages 5-pp, 2006.

[17] Jin Rencheng, Wang Hongbin, Peng Bo, and Ge Ning. Research on rssi-based localization in wireless sensor networks. pages 1-4, 2008. 
[18] Paramvir Bahl and Venkata N Padmanabhan. Radar: An in-building rf-based user location and tracking system. 2:775-784, 2000.

[19] Frank Vanheel, Jo Verhaevert, Eric Laermans, Ingrid Moerman, and Piet Demeester. Automated linear regression tools improve rssi wsn localization in multipath indoor environment. EURASIP Journal on Wireless Communications and Networking, 2011(1):1-27, 2011.

[20] Yingying Chen, J-A Francisco, Wade Trappe, and Richard P Martin. A practical approach to landmark deployment for indoor localization. In Sensor and Ad Hoc Communications and Networks, 2006. SECON'O6. 2006 3rd Annual IEEE Communications Society on, volume 1, pages 365-373. IEEE, 2006.

[21] I. Hen. Mimo architecture for wireless communication. Intel Technology Journal, 10(2):157-165, 2006.

[22] Thomas Eng, Ning Kong, and Laurence B Milstein. Comparison of diversity combining techniques for rayleigh-fading channels. Communications, IEEE Transactions on, 44(9):1117-1129, 1996.

[23] Theodore S Rappaport et al. Wireless communications: principles and practice, volume 2. Prentice Hall PTR New Jersey, 1996.

[24] Amitabha Ghosh, Amar H Patel, and Chellury Ram Sastry. Radio frequency based indoor localization in wireless sensor networks. 